\title{
Scoping review: práticas corporais na atenção básica em saúde ${ }^{1}$
}

\section{Scoping review: bodily practice in primary health care \\ Scoping review: prácticas corporales in atención primaria de salud}

\author{
iD) Ana Paula Dahlke \\ Universidade Federal do Rio Grande do Sul, Porto Alegre, Rio Grande do Sul, Brasil \\ anapauladahlke@hotmail.com \\ iD (9) Fabiana Fernandes Vaz \\ fabianavaz@gmail.com
}

Resumo: As práticas corporais (PC) foram introduzidas no âmbito da saúde pública pela Política Nacional de Promoção da Saúde, em 2006. Utilizando o método scoping review, objetivamos investigar as PC propostas na Atenção Básica, de 2006 a 2015. Selecionamos 24 estudos dentre 1168 resgatados pela busca inicial. As PC mais relatadas foram a dança, o alongamento, as práticas orientais e a caminhada e, a maioria, foi escoIhida de acordo com a experiência do profissional que conduziu a atividade. Os locais utilizados foram as unidades de saúde e arredores. Parte das intervenções foram conduzidas por estagiários e o profissional de educação física teve a participação mais preeminente nas intervenções.

Palavras-chave: Práticas Corporais; Atenção Primária à Saúde; Promoção da Saúde; Sistema Único de Saúde; Exercício.

\begin{abstract}
Bodily practice (BP) were introduced in the scope of public health by the National Health Promotion Policy in 2006. Using the scoping review method, we aimed to investigate BPs proposed in Primary Care from 2006 to 2015. We selected 24 studies out of 1168 rescued
\end{abstract}

10 presente trabalho não contou com apoio financeiro de nenhuma natureza para sua realização 
by the initial search. The most reported BP were dance, stretching, oriental practices and walking and, most, were chosen according to the professional experience that led the activity. The places used were the health facilities and surroundings. Some of the interventions were conducted by trainees and the physical education professional had the most pre-eminent participation in the interventions.

Keywords: Primary Health Care; Health Promotion; Unified Health System; Exercise.

Resumen: Las prácticas corporales (PC) fueron introducidas en el ámbito de la salud pública por la Política Nacional de Promoción de la Salud, en 2006. Utilizando el método scoping review, objetivamos investigar las PC propuestas en la Atención Básica, de 2006 a 2015. Seleccionamos 24 estudios de entre 1168 rescatados por la búsqueda inicial. Las PC más relatadas fueron la danza, el estiramiento, las prácticas orientales y la caminata y, la mayoría, fue elegida de acuerdo con la experiencia del profesional que condujo la actividad. Los lugares utilizados fueron las unidades de salud y los alrededores. Parte de las intervenciones fueron conducidas por pasantes y el profesional de educación física tuvo la participación más preeminente en las intervenciones.

Palabras clave: Atención Primaria de Salud; Promoción de la Salud; Sistema Único de Salud; Ejercicio.

Submetido em: 12/09/2018

Aceito em: 23/09/2019 


\section{Introdução}

Saúde e atividade física possuem uma conexão muito íntima. Por muito tempo, essa conexão se deu por meio de conteúdos biológicos, com entendimento de que bastaria movimentar o corpo para ter saúde. A partir da Reforma Sanitária e de um novo entendimento do conceito de saúde (conceito ampliado), o conceito de práticas corporais (PC) foi introduzido. Apesar de polissêmico, quando relacionado à atividade física e saúde, pode ser entendido como uma forma de valorizar a atividade e o praticante, considerando os sentidos e significados, entendendo o movimento corporal como parte da cultura de uma comunidade. Movimentar-se não traz só benefícios para o corpo físico. O movimento corporal aliado à cultura, ao prazer, ao lazer, aos interesses e necessidades individuais e coletivas tem potencial para construir relações de afeto, de cuidado, de autonomia e valorização da saúde.

O conceito de PC não representa um sinônimo de atividade física. Trata-se de uma valorização do significado das atividades físicas para o seu praticante (FRAGA et al., 2013). Para Carvalho (2007), existe uma contraposição à ideia de atividade física, à medida que a última homogeneíza o coletivo, padroniza e nivela o corpo, com base na racionalidade biomédica, enquanto que PC, para a mesma autora, é a manifestação da cultura corporal de um grupo embasada nos significados que as pessoas Ihes atribuem. "Contemplam as vivências lúdicas e de organização cultural e operam de acordo com a lógica do acolhimento, aqui no sentido de estar atento às pessoas, de trabalhar ouvindo seus desejos e necessidades e, ao mesmo tempo, orientá-las e encaminhá-las de modo a atendê-las para além do imediato". (CARVALHO, 2007, p. 65).

Carvalho (2006) ainda traz que as PC podem ampliar as possibilidades de encontrar, escutar, observar e mobilizar as pessoas adoecidas para que nesse processo possam construir relações de vínculo, de corresponsabilidade, relações autônomas, inovadoras e socialmente inclusivas, valorizando o uso dos espaços públicos. 
Nesse sentido, e sem deixar de considerar as questões emergentes relacionadas à saúde pública e ao crescimento das doenças crônico-degenerativas, a Política Nacional de Promoção da Saúde (PNPS), criada em 2006, busca contemplar as populações para além dos muros dos serviços de saúde, propondo incidir sobre as condições de vida e favorecer a ampliação de escolhas saudáveis, tendo como objetivo promover a qualidade de vida e reduzir a vulnerabilidade e riscos à saúde relacionados aos seus determinantes (BRASIL, 2010). Foi por meio da PNPS, que as PC foram formalmente introduzidas na Atenção Básica em Saúde (ABS).

ABS se caracteriza por ser a porta de entrada principal do Sistema Único de Saúde (SUS). É realizado nesse espaço um conjunto de ações de saúde, no âmbito individual e coletivo, que abrange a promoção e a proteção da saúde, a prevenção de agravos, o diagnóstico, o tratamento, a reabilitação, a redução de danos e a manutenção da saúde com o objetivo de desenvolver uma atenção integral que impacte positivamente na saúde das coletividades. Fazem parte da ABS, as Unidades Básicas de Saúde (UBS) e as Unidades com Estratégia da Saúde da Família (ESF) com o apoio dos Núcleos de Apoio à Saúde da Família (NASF), a Academia da Saúde e os Consultórios na Rua.

O NASF tem como objetivo ampliar a abrangência, a resolutividade, a territorialização, a regionalização, bem como a ampliação das ações da ABS (BRASIL, 2010), possibilitando maior capacidade de resposta das ESF aos problemas da população.

Um NASF deve ser constituído por uma equipe, na qual profissionais de diferentes áreas do conhecimento atuam em conjunto com os profissionais das equipes de Saúde da Família, compartiIhando e apoiando as práticas em saúde nos territórios sob responsabilidade das equipes de Saúde da Família (BRASIL, 2009). As ações do NASF são realizadas a partir de demandas identificadas no trabalho em conjunto com as equipes ESF e/ou Academia da Saúde (BRASIL, 2012).

Os NASF devem utilizar as Academias da Saúde como espaços que ampliam a capacidade de intervenção coletiva das equipes 
para as ações de promoção da saúde, buscando fortalecer o protagonismo de grupos sociais em condições de vulnerabilidade na superação de suas condições (BRASIL, 2012).

O Programa Academia da Saúde foi instituído no âmbito do SUS com o objetivo de contribuir para a promoção da saúde da população a partir da implantação de polos com infraestrutura, equipamentos e quadro de pessoal qualificado para a orientação de PC, atividades físicas e práticas de lazer, oportunizando modos de vida saudáveis.

A criação dos NASF e das Academias da Saúde ampliou a perspectiva do atendimento integral, com base nas ações específicas priorizadas pela PNPS, que incluiu a prática corporal em intervenções na rede básica de saúde.

Devido à importância da inserção dessas práticas nos serviços de ABS, o objetivo do presente trabalho é investigar as propostas de intervenção com PC realizadas pelas equipes multiprofissionais no contexto da ABS no Brasil.

\section{Metodologia}

Para dar conta do objetivo da pesquisa, utilizamos o método scoping review, que propõe mapear a literatura existente em um campo de interesse em termos de volume, examinar a extensão, alcance e natureza da atividade de pesquisa em determinada área temática (ARKSEY; O'MALLEY, 2005).

A pesquisa foi implementada a partir janeiro de 2016 em quatro bases de dados eletrônicas: Scielo, Bireme, Bases de teses e dissertações da Capes e Google Acadêmico. As buscas foram feitas a partir do ano de 2006, tendo como base a implantação da PNPS, que insere as PC no campo da saúde, até dezembro de 2015.

Seguimos a recomendação de Arksey e O'Malley (2005) de manter uma abordagem ampla de pesquisa, e para isso utilizamos os 
termos: "práticas corporais"; "atenção básica"; "atenção primária"; "academia da saúde"; "NASF"; "SUS"; "unidade básica de saúde", e "ESF". Os operadores lógicos and e or foram usados para combinar os descritores e termos utilizados na busca pelos trabalhos.

Foram observadas as referências bibliográficas dos trabaIhos selecionados, buscando novas possibilidades com a temática proposta.

Foram incluídos artigos, teses, dissertações, monografias de conclusão de curso de especialização e monografias de conclusão de curso de graduação.

As publicações selecionadas deveriam estar disponíveis integralmente on-line, sem qualquer cobrança para o download do material. Publicações em línguas estrangeiras foram rejeitadas. Foram incluídos os trabalhos de intervenção, estudos que descrevem um trabalho de intervenção ou trabalhos que realizaram entrevistas com as equipes responsáveis pelas intervenções. Os trabalhos que utilizaram PC como sinônimo de atividade física, entendendo esse conceito de forma estritamente biologicista, ou de causa e efeito, sem caracterizar as práticas como uma forma de cuidado integral à saúde foram excluídos do presente estudo.

Publicações que apresentavam os mesmos dados, por exemplo: uma tese e um artigo extraído desta mesma tese, apenas a publicação mais completa foi incluída nesta pesquisa.

A pesquisa foi realizada em dois momentos. No primeiro momento, foi realizada a leitura do título e o resumo, respeitando os critérios de inclusão. Num segundo momento, foi realizada a leitura completa da publicação, avaliando se cumpria os critérios de eleição.

Considerando todas as fontes de busca foram identificados 1168 estudos. Com base na leitura dos títulos e resumos, 78 estudos foram selecionados. Dos 78 artigos que foram lidos na íntegra, 22 foram selecionados para análise final. Pela análise das referências bibliográficas, foram selecionados dois trabalhos, totalizando 24. 
Primeiramente foi dada especial atenção à análise numérica, natureza e distribuição dos estudos incluídos na revisão. Em segundo lugar, a literatura foi organizada tematicamente, de acordo com os tipos diferentes de intervenção, profissionais envolvidos, público atendido e locais onde as práticas aconteceram.

\section{As práticas corporais propostas na Atenção Básica em Saúde}

No que diz respeito às atividades desenvolvidas, as mais citadas foram: dança e variações como dança circular e biodança, práticas de alongamento e relaxamento e a caminhada orientada. As Práticas Integrativas e Complementares (PICs), mais relacionadas às $P C$ orientais também foram citadas.

Os esportes e os jogos, na maioria das vezes não foram exemplificados, quando citados: tênis de mesa, futsal, voleibol, basquete, futebol de areia e futebol. Foram citados também o treinamento de força, as ginásticas e a Capoeira. Outras atividades foram citadas. 
Tabela 1: Atividades desenvolvidas

\begin{tabular}{|c|c|}
\hline Atividades desenvolvidas & Referências \\
\hline Danças e variações & $\begin{array}{l}\text { CARVALHO; FREITAS, 2006; MORAIS, 2009; MENDES, 2010; BEZERRA, } \\
\text { 2011; MENDONÇA, 2012; RODRIGUES et al. 2012; VUADEN, 2012; MEN- } \\
\text { DES, 2013; NASCIMENTO, 2013; CONCEIÇÃO; MORAES; FRANCHINI, } \\
\text { 2014; SKOWRONSKI, } 2014\end{array}$ \\
\hline $\begin{array}{l}\text { Práticas de alongamento e } \\
\text { relaxamento }\end{array}$ & $\begin{array}{l}\text { CARVALHO; FREITAS, 2006; ALMEIDA, 2009; MORAIS, 2009; TORRES, } \\
\text { 2011; GUILIANO, 2012; MENDONÇA, 2012; VUADEN, 2012; MEN- } \\
\text { DES, 2013; CONCEIÇÃO; MORAES; FRANCHINI, 2014; DAHLKE, 2014; } \\
\text { SKOWROSKI, } 2014\end{array}$ \\
\hline $\begin{array}{l}\text { Caminhada } \\
\text { orientada }\end{array}$ & $\begin{array}{l}\text { CARVALHO; FREITAS, 2006; WARSCHAUER, 2007, 2008; } \\
\text { SILVA et al., 2009; CARNEIRO, 2010; MENDES, 2010; } \\
\text { MENDONÇA, 2012; MENDES, 2013; CONCEIÇÃO; MORAES; FRANCHINI, } \\
\text { 2014; DAHLKE, 2014; SKOWROSKI, } 2014\end{array}$ \\
\hline Lian Gong & NASCIMENTO, 2013 \\
\hline Yoga & CÉSAR, 2012 \\
\hline Tai Chi Chuan I e & CARNEIRO, 2010 \\
\hline Tai Chi Pai Lin & WARSCHAUER, 2008 \\
\hline Lian Gong & SKOWRONSKI, 2014 \\
\hline Qi Gong & MORAIS, 2009 \\
\hline Lien Chi & MENDES, 2010 \\
\hline $\begin{array}{l}\text { Esportes (tênis de mesa, } \\
\text { futsal, voleibol, basquete, } \\
\text { futebol de areia e futebol) }\end{array}$ & MENDONÇA, 2012; SKOWRONSKI, 2014; ABIB; DAMICO, 2013 \\
\hline Treinamento de força & $\begin{array}{l}\text { WARSCHAUER, 2008; ALMEIDA, 2009; GIULIANO, 2012; MENDONÇA, } \\
\text { 2012; NASCIMENTO, 2013; DAHLKE, 2014; SKOWROSKI, } 2014\end{array}$ \\
\hline Ginásticas & $\begin{array}{l}\text { CARVALHO; FREITAS, 2006; MENDONÇA, 2012; RODRIGUES, 2012; } \\
\text { SKOWROSKI, } 2014\end{array}$ \\
\hline Capoeira & ALMEIDA, 2009; FREITAS, 2012; SKOWROSKI, 2014 \\
\hline Pilates & SKOWROSKI, 2014 \\
\hline Unibiótica & CARNEIRO, 2010 \\
\hline Expressão corporal & MARCONDES, 2007; MORAIS, 2009 \\
\hline Atividades lúdicas & MENDES, 2010; GORSKI; PEREIRA, 2013 \\
\hline
\end{tabular}

Fonte: Elaborada pelas autoras, 2018. 


\section{Motivos para escolha das práticas corporais na Atenção Básica em Saúde}

Dos motivos identificados para a escolha das PC pode-se destacar a formação e identificação do profissional que conduz a atividade. Ou seja, nas intervenções relatadas a formação, os cursos, as vivências pessoais e os interesses do profissional foram fatores determinantes para a escolha da prática a ser implementada (WARSCHAUER et al., 2007; MARCONDES, 2007; WARSCHAUER, 2008).

Alguns trabalhos relataram a escolha da PC baseada nas atividades que as secretarias de saúde dos municípios, nos quais foram realizadas as pesquisas, elegem como prioritárias, fomentando e promovendo capacitação aos profissionais interessados em multiplicar as práticas nas unidades de saúde (WARSCHAUER, 2008; MONTEIRO, 2013).

A praticidade e a baixa complexidade também foram consideradas na escolha das PC, privilegiando atividades que não necessitam de materiais ou locais específicos para a prática ou a especialização de profissionais, evitando expor os usuários a riscos de lesões ou outros agravos. Assim, a caminhada, as danças, as práticas orientais, alongamento e relaxamento, por serem atividades simples, que não necessitam de muitos recursos, fáceis de condução em grupos heterogêneos foram as principais atividades identificadas (WARSCHAUER et al., 2007).

A segurança dos usuários também foi trazida em alguns estudos, relatando a estrutura precárias de alguns municípios e regiões, além da violência urbana. Foram citadas as ruas não asfaltadas, vias sem manutenção, falta de calçadas, avenidas muito movimentadas, ruas muito íngremes, criminalidade, entre outros (FREITAS, 2012; MENDONÇA, 2012; VUADEN, 2012; WARSCHAUER, 2008). 
Dois trabalhos relataram buscar PC que se identificassem com o grupo, numa tentativa de contemplar os usuários envolvidos, privilegiando os conhecimentos e práticas já presentes no grupo ou na comunidade (DAHLKE, 2014 e MENDES, 2013), e um terceiro trabalho relatou investigar a demanda do local para planejar as práticas ofertadas (MARCONDES, 2007).

O SUS traz em seus princípios a prática do controle social. Essa ferramenta permite ao usuário do sistema participar das decisões "seja nos conselhos locais, distritais e de unidades, seja nas conferências de saúde, possibilita a organização de vários grupos comunitários interagindo com as temáticas relativas à sua realidade" (BRASIL, 2009, p. 127). Essa participação popular também pode e deve ser aplicada na construção das PC, possibilitando definir as atividades que expressem as necessidades e desejos, considerando a contribuição coletiva e as atividades que já ocorrem na comunidade, evitando imposições de ações e modelos por parte do profissional e a falta de identificação dos sujeitos com as atividades propostas.

Portanto, o planejamento participativo, apesar de conter aspectos de difícil controle em sua materialização, é uma estratégia agregadora e fortalecedora de laços entre o profissional e a comunidade, fundamental para o sucesso de qualquer atividade (BRASIL, 2009, p.127).

Assim, as práticas devem ser construídas a partir de componentes culturais, históricos, políticos, econômicos e sociais do local e cabe ao profissional a leitura abrangente do contexto onde irá atuar profissionalmente e como ator social (BRASIL, 2009).

Freitas (2012) e Dahlke (2014) relataram a participação de usuários com vivências em alguma prática corporal, oportunizando a troca, a construção e o compartilhamento de saberes como meio de produção de vida, corresponsabilidade, autonomia e integração, o que corresponde com a proposta colocada pelas diretrizes do NASF (BRASIL, 2009). 


\section{Usuários atendidos}

As PC no âmbito da ABS, de acordo com os trabalhos selecionados, foram ofertadas para todos interessados em participar, tendo como única prerrogativa fazer parte do território atendido pela unidade de saúde. Não obstante, algumas unidades ofereceram atividades para grupos específicos, formados com base no diagnóstico de alguma patologia, como por exemplo, grupo para hipertensos, grupos para diabéticos, obesos, gestantes, idosos, pessoas da comunidade com algum déficit físico ou mental (RODRIGUES et al., 2012; FREITAS, 2012). A divisão em grupos delimitados por patologias pode enfatizar mais a doença e menos os sujeitos envolvidos e suas necessidades, que vão além do corpo físico e doente, bem como limitar a compreensão do processo saúde-doença-intervenção e afetar negativamente a produção de saúde (CARVALHO, 2006; FREITAS; CARVALHOS; MENDES, 2013).

A participação mais predominante identificada foi de mulheres acima de 40 anos de idade (SILVA et al., 2009; BEZERRA, 2011; GIULIANO, 2012; MENDONÇA, 2012; MENDES, 2013; DAHLKE, 2014; CONCEIÇÃO; MORAES; FRANCHINI, 2014; SKOWRONSKI, 2014) e de pessoas idosas ou da terceira idade, sem distinção de sexo e gênero (WARSCHAUER, 2008; VUADEN, 2012 e NASCIMENTO, 2013).

A participação de jovens e adultos nas unidades de saúde foi muito inferior à participação de idosos. Um dos motivos para a baixa adesão desses grupos nos projetos propostos pode estar relacionada ao horário das atividades, geralmente em horário comercial e justamente quando essas populações estão envolvidas em atividades laborais e/ou escolares/acadêmicas (MARCONDES, 2007; MENDONÇA, 2012). 


\title{
Locais onde aconteceram as práticas corporais
}

\begin{abstract}
A falta de uma estrutura adequada para a realização das PC nas unidades de saúde implica na busca por outros locais disponíveis e de fácil acesso para a comunidade que deseja participar das atividades.

Em alguns trabalhos, as práticas foram realizadas dentro dos espaços das UBS e ESF ou ainda em locais próximos às unidades inseridos no território. As praças e parques (quando existentes) também foram usados como espaços para o encontro dos grupos, usuários e profissionais, bem como o espaço das escolas próximas.

Os salões comunitários, associação de moradores, salões paroquiais das igrejas locais foram exemplos de espaços cedidos pela comunidade para que as práticas pudessem acontecer. Houve ainda encontros realizados no pátio em frente à Unidade de Saúde, assim como na casa de um dos participantes do grupo.
\end{abstract}

Tabela 2: Locais de Práticas

\begin{tabular}{|l|l|}
\hline Locais de prática & Referências \\
\hline Dentro das UBS e ESF & $\begin{array}{l}\text { CARVALHO; FREITAS, 2006; WARSCHAUER et al., 2007; WARSCHAUER, 2008; } \\
\text { MORAIS, 2009; SILVA; 2009; CARNEIRO, 2010; MARCONDES, 2010; FREITAS, } \\
\text { 2012; GIULIANO, 2012; RODRIGUES et al., 2012; VUADEN, 2012; GORSKI; } \\
\text { PEREIRA, 2013; NASCIMENTO, 2013; DAHLKE, 2014 }\end{array}$ \\
\hline $\begin{array}{l}\text { Proximidades das UBS } \\
\text { e ESF }\end{array}$ & MENDONÇA, 2012; MENDES, 2010 \\
\hline Praças e parques & MENDONÇA, 2012; ABIB; DAMICO, 2013; MENDES, 2013 \\
\hline Escolas & FREITAS, 2012; TORRES, 2012 \\
\hline $\begin{array}{l}\text { Espaços cedidos pela } \\
\text { comunidade }\end{array}$ & $\begin{array}{l}\text { WARSCHAUER, 2008; BEZERRA, 2011; VUADEN 2012; CONCEIÇÃO; MORAES; } \\
\text { FRANCHINI, 2014 }\end{array}$ \\
\hline Residência de usuário & CÉSAR, 2012 \\
\hline
\end{tabular}

Fonte: Elaborada pelas autoras, 2018. 


\section{Profissionais que estão interessados em falar sobre prá- ticas corporais na Atenção Básica em Saúde}

Dentre os trabalhos selecionados, diferentes profissionais estiveram envolvidos na confecção dos mesmos. Os profissionais da educação física são os que atuam diretamente com as PC na atenção básica e na produção do conhecimento não foi diferente. Dezenove profissionais da educação física redigiram estudos com essa temática, seguidos de cinco profissionais da enfermagem, dois da fisioterapia, dois da terapia ocupacional, dois da psicologia e um da farmácia, um da medicina e um da odontologia.

Desses estudos, dez foram artigos publicados em revistas nacionais, quatro dissertações de mestrado na área da Educação Física, um da Saúde Coletiva e um da Enfermagem. Além de cinco trabalhos de conclusão de curso de graduação em Educação Física, sendo quatro licenciatura e um bacharelado. Mais um trabalho de conclusão de curso de especialização em Atenção Básica em Saúde da Família e uma tese de doutorado em Educação Física.

\section{Profissionais que propuseram práticas corporais na Atenção Básica em Saúde}

Nos trabalhos selecionados para compor o presente estudo, 0 profissional da educação física apareceu como proponente das PC na atenção básica em 15 dos 24 trabalhos. Apesar de não ser um campo exclusivo de atuação desse profissional, foi pela introdução das PC e das atividades físicas na PNPS, que o profissional de educação física ganhou espaço na área da saúde.

Outros profissionais também apareceram como condutores de PC: médicos (4); psicólogos (4); fisioterapeutas (3); terapeutas ocupacionais (3); fonoaudiólogo (1) e um relato sobre a residência multiprofissional em saúde. 
A presença de acadêmicos dos cursos de educação física, fisioterapia e terapia ocupacional em períodos de estágio também foi citada (CARVALHO; FREITAS, 2006; WARSCHAUER et al., 2007; WARSCHAUER, 2008; TORRES, 2011; FREITAS, 2012; RODRIGUES et al., 2012; VUADEN, 2012; DAHLKE, 2014). Desses, três trabalhos trazem relatos de estagiários do Programa de Educação pelo Trabalho para a Saúde PET-Saúde (PET-Saúde). O PET-Saúde, vinculado ao Programa Nacional de Reorientação da Formação Profissional em Saúde (Pró-Saúde), tem como objetivo estimular grupos de aprendizagem tutorial na ESF. É uma ferramenta importante para a formação dos profissionais que pretendem atuar na área da saúde, buscando melhorias no atendimento à população e na operacionalização do SUS, através da cooperação entre gestores do SUS e as universidades, incorporando à formação profissional saberes sobre o processo saúde-doença, além de ampliar as práticas educacionais na rede de atenção básica à saúde.

Silva et al. (2009) destacaram dificuldade em envolver a equipe multiprofissional e ampliar os encontros e os saberes compartilhados devido à grande demanda do serviço e a diferença nos horários de trabalho dos diversos profissionais. É importante destacar que o trabalho em equipes multiprofissionais possibilita a realização do atendimento integral e articulação dos saberes e disciplinas necessárias para o atendimento em saúde (FRANCIONI; SILVA, 2007). A atenção integral faz parte de um processo de mudanças nas relações de poder entre os profissionais da saúde, buscando a formação efetiva de equipes multiprofissionais, interdisciplinares e na relação entre profissionais de saúde e usuários, permitindo que os usuários tenham autonomia para "serem sujeitos da sua saúde e não pacientes da ação dos profissionais" (BRASIL, 2004, p. 8).

Conceição, Moraes e Franchini (2014) fazem um relato da experiência de atuação interdisciplinar, composta pela fisioterapia, terapia ocupacional e educação física, na produção do cuidado na ABS utilizando as PC como recurso. Segundo os autores, a atuação neste modelo possibilita uma ampliação do olhar sobre os indivíduos e sobre o território, garantindo um cuidado integral em saúde. 
Gorski e Pereira (2013) relatam que, apesar da experiência positiva, tiveram dificuldades em relação ao trabalho interdisciplinar, pois essa prática nem sempre foi alcançada nos diversos campos e profissionais da saúde. As dificuldades no/do trabalho em equipe, a troca de experiências e a convivência com diferentes estilos de pensar, foram questões que permearam o trabalho dos autores. No entanto, o trabalho interdisciplinar desenvolvido pela educação física, fonoaudiologia e psicologia, conforme relatam os autores, mostrou-se enriquecedor, contribuindo para o processo de formação ampla dos sujeitos, no que diz respeito à integração das crianças (envolvidas no estudo) com as três profissões.

Além da caracterização dos profissionais envolvidos nas publicações dos trabalhos, fizemos o levantamento de acordo com as regiões brasileiras que construíram os estudos selecionados para esta revisão. A região sudeste é a que mais tratou do tema, seguida pela região sul e demais regiões. Um dos trabalhos foi construído utilizando entrevistas com profissionais que atuam nas Academias da Saúde nos mais diversos estados brasileiros.

\section{Tabela 3: Regiões}

\begin{tabular}{|l|l|}
\hline Regiões & Referências \\
\hline Sudeste & CARVALHO; FREITAS, 2006; MARCONDES, 2007; WARSCHAUER et al., 2007; WA- \\
& RSCHAUER, 2008; ALMEIDA, 2009; CARNEIRO, 2010; FREITAS, 2012; MENDES, \\
& 2013; CONCEIÇÃO; MORAES; FRANCHINI, 2014 \\
\hline Sul & TORRES, 2011; CÉSAR, 2012; GIULIANO, 2012; MENDONÇA, 2012; VUADEN, \\
& 2012; ABIB; DAMICO, 2013; GORSKI.; PEREIRA, 2013; DAHLKE, 2014 \\
\hline Nordeste & SILVA et al., 2009; MENDES, 2010; BEZERRA, 2011; RODRIGUES et al., 2012 \\
\hline Norte & MORAIS, 2009; NASCIMENTO et al., 2013 \\
\hline Nacional & SKOWRONSKI, 2014 \\
\hline
\end{tabular}

Fonte: Elaborada pelas autoras, 2018.

\section{Tipos de estudo}

Nos trabalhos incluídos foram encontrados diferentes tipos de estudos, alguns envolveram entrevistas, entrevistas semiestru- 
turadas, observação, intervenção, observação participante, exploratório-descritivo e grupo focal.

\section{Tabela 4: Tipos de Estudos}

\begin{tabular}{|l|l|}
\hline Tipos de estudos & Referências \\
\hline Entrevista & $\begin{array}{l}\text { MARCONDES, 2007; WARSCHAUER, 2008; CÉSAR, 2012; FREITAS, 2012; } \\
\text { MENDONÇA, 2012; SKOWRONSKI, 2014 }\end{array}$ \\
\hline Entrevista-semiestruturada & CARNEIRO, 2010; BEZERRA, 2011; CÉSAR, 2012; GORSKI; PEREIRA, 2013 \\
\hline Observação & $\begin{array}{l}\text { MARCONDES, 2007; WARSCHAUER et al., 2007; WARSCHAUER, 2008; } \\
\text { MORAIS, 2009; FREITAS, 2012; MENDONÇA, 2012 }\end{array}$ \\
\hline Intervenção & $\begin{array}{l}\text { CARVALHO; FREITAS, 2006; MARCONDES, 2007; SILVA et al., 2009; } \\
\text { TORRES, 2011; RODRIGUES et al., 2012; VUADEN, 2012; ABIB; DAMICO, } \\
\text { 2013; CONCEIÇÃO, et al., 2014; DAHLKE, 2014 }\end{array}$ \\
\hline Observação participante & CARVALHO; FREITAS, 2006; MENDES, 2013 \\
\hline Exploratório-descritivo & MENDES, 2010; BEZERRA, 2011; GIULIANO, 2012; GUARDA, 2014 \\
\hline Grupo focal & GIULIANO, 2012 \\
\hline
\end{tabular}

Fonte: Elaborada pelas autoras, 2018.

Dos nove trabalhos que realizam a intervenção como parte da metodologia de pesquisa, todos foram escritos por um profissional de educação física ou este profissional compõem o grupo de pesquisadores que escreveu o estudo. E dos nove estudos, seis estão relacionados a estagiários que realizaram estágio na Atenção Básica, sendo três vinculados ao PET-Saúde e um à residência multiprofissional.

Por fim, César (2012) relatou a sua preocupação em relação a continuidade das ações proposta, pois seria interessante que a comunidade pudesse desfrutar das PC independentemente de quem a ministrasse. $O$ autor relata que após a sua saída, a atividade deixou de ser realizada.

Observando os estudos que compuseram a presente revisão, a grande maioria foi proposta por estudantes em seu período de estágio (curricular ou não). Portanto, essas atividades foram ofertadas somente durante a presença do estagiário. Dessa forma, salientamos a importância da autonomia da comunidade nas pro- 
postas e intervenções em saúde, oportunizando que as PC possam ser continuadas pelos usuários e pela comunidade envolvida. Ou seja, quanto mais os sujeitos estiverem comprometidos com seus projetos de saúde e no controle social da atenção em saúde e do SUS, mais estarão aptos a serem protagonistas de seus projetos de vida e saúde.

\section{Considerações Finais}

As PC no âmbito da atenção básica em saúde possuem características capazes de mobilizar os indivíduos e a comunidade, criando laços de afeto e corresponsabilidade não só entre os sujeitos, mas também entre os sujeitos e os profissionais da saúde, a comunidade e os espaços públicos que podem ser ocupados para a produção de saúde.

O trabalho com PC nos ambientes de saúde deve englobar a cultura local, o desejo dos participantes, a pluralidade do grupo e, ainda, oferecer práticas diversificadas, permitindo aos usuários a possibilidade de se identificar com alguma delas e adotá-la em seu dia a dia, não somente como uma prática de cuidado em saúde, mas como uma prática de prazer, com sentidos e significados que não se limitam no corpo físico, mas transpassam para a totalidade do que é o sujeito e tudo que ele representa, permitindo-lhe conhecer as capacidades e limites do seu próprio corpo.

Há um número ilimitado de PC que podem ser trabalhadas nos ambientes de saúde, principalmente se levarmos em consideração a cultura e as construções sociais de cada comunidade. Portanto, não há a necessidade de se fixar nas atividades de modelos prontos ou aquelas incessantemente divulgadas pela mídia como práticas saudáveis e com resultados confiáveis. Ou ainda, ofertar práticas de acordo com a vontade do profissional, por ser uma prática que ele melhor domina. A comunidade deve ser parte integrante das escolhas, participar das decisões e suas necessida- 
des e desejos devem ser respeitados, tendo seus conhecimentos e vivências aproveitados para serem compartilhados pela comunidade, contribuindo para a autonomia e a criação de novas práticas e saberes em saúde.

As práticas mais relatadas nos trabalhos selecionados referem-se à dança, ao alongamento, às PIC, à caminhada, aos jogos e esportes. São, no geral, atividades de baixa complexidade, que não exigem materiais e espaços muito elaborados, podendo ser executadas por diferentes públicos e em locais diversos, pois não demandam muitos recursos.

Observamos que parte da oferta de PC é feita por estagiários. Isso se traduz numa atividade com tempo determinado para começar e terminar. Essa situação cria uma demanda por práticas que nem sempre pode ser suprida pelos profissionais existentes na atenção básica. Por isso é tão importante que os saberes que circulam dentro da comunidade sejam compartilhados, permitindo que as práticas possam continuar acontecendo independente da permanência do estagiário ou do profissional. Tanto os estagiários quanto os profissionais podem contribuir para o aprimoramento das práticas locais, pois ao mesmo tempo que ampliam suas vivências, ampliam também as vivências da comunidade dos usuários, desenvolvendo relações autônomas e não de dependência.

Os profissionais da educação física são os que mais atuaram com PC na atenção básica de acordo com os trabalhos resgatados pela presente revisão, e esse fato é compreensível, uma vez que as PC em geral fazem parte do seu repertório acadêmico e profissional. Contudo, a atuação e intervenção em equipes multiprofissionais pode enriquecer a troca de saberes e a produção do conhecimento, além de proporcionar um cuidado em saúde integral e globalizado, com olhares mais abrangentes beneficiando amplamente os usuários dos serviços de saúde.

As PC foram introduzidas na atenção básica formalmente a partir do ano de 2006. Considerando que as políticas públicas em saúde são processos que demandam um longo prazo, ainda há 
muito para se fazer na atenção básica. A integração dos profissionais entre si e com as comunidades é o primeiro passo para o sucesso das relações e das práticas em saúde. A ocupação dos espaços públicos e a ampliação dos espaços destinados ao lazer das comunidades é outra questão que merece atenção. A formação de profissionais preparados para lidar com os diferentes níveis de adoecimento e de sofrimento merece destaque, além de profissionais que entendam e saibam valorizar os saberes que circulam nas comunidades, sabendo aproveitá-los para a construção de novos saberes baseados na cultura de paz e saúde.

\section{Referências bibliográficas}

ABIB, L. T.; DAMICO, J. G. S.. Possibilidades de composição de uma equipe de consultório na rua. Revista Didática Sistêmica. Rio Grande, Universidade Federal do Rio Grande. Edição Especial (2013), p. 97-111, 2013.
ALMEIDA, P. Núcleo de Apoio as Equipes de Saúde da Família
(NASF): Uma breve reflexão. 2009. 53 f. Trabalho de Conclusão de Curso (Curso de Especialização em Atenção Básica em Saúde da Família), Universidade Federal de Minas Gerais, Guanhães, 2009. Disponível em: https://www.nescon.medicina.ufmg.br/ biblioteca/imagem/2261.pdf. Acesso em: 27 jan. 2016.

BEZERRA, I. M. P. Estratégias ou táticas alternativas: procurando novos caminhos para a promoção da saúde entre modelos assistenciais e processos de trabalho. 2011. 143 f. Dissertação de Mestrado (Mestrado do Centro de Ciências Exatas e da Natureza) - Programa de Pós-graduação em Modelos de decisão e saúde, Universidade Federal da Paraíba, João Pessoa, 2011. Disponível em: http://bdtd.biblioteca.ufpb.br/tde_arquivos/31/TDE-201103-01T133823Z-864/Publico/arquivototal.pdf. Acesso em: 29 jan. 2016. 
BRASIL. Ministério da Saúde. Secretaria de Gestão do Trabalho e da Educação na Saúde. Departamento de Gestão da Educação na Saúde. AprenderSUS: o SUS e os cursos de graduação da área da saúde. Brasília, 2004.

BRASIL. Ministério da Saúde. Secretaria de Vigilância em Saúde. Secretaria de Atenção à Saúde. Política Nacional de Promoção da Saúde. Brasília, 2006.

BRASIL. Ministério da Saúde. Portaria n 719, de 7 de abril de 2011. Institui o Programa Academia da Saúde no âmbito do Sistema Único de Saúde. 2011

BRASIL. Ministério da Saúde. Secretaria-Executiva. Secretaria de Vigilância em Saúde. Glossário temático: promoção da saúde. Brasília, 2012.

BRASIL. Ministério da Saúde. Secretaria de Atenção à Saúde. Departamento de Atenção Básica. Diretrizes do NASF: Núcleo de Apoio a Saúde da Família. Brasília, 2009.

BRASIL. Ministério da Saúde. Secretaria de Vigilância em Saúde. Secretaria de Atenção à Saúde. Política Nacional de Promoção da Saúde. Brasília, 3 ed., 2010.

BRASIL. Ministério da Saúde. Secretaria de Atenção à Saúde. Departamento de Atenção Básica. Política Nacional de Atenção Básica. Brasília, 2012.

CARNEIRO, A. C. L. L. Práticas educativas nas unidades básicas de saúde de Belo Horizonte e sua relação com a promoção da saúde. 2010. 128 f. Dissertação de Mestrado (Mestrado da Escola de Enfermagem), Universidade Federal de Minas Gerais, Belo Horizonte, 2010. Disponível em: http://www.enf.ufmg.br/pos/ defesas/643M.PDF. Acesso em: 28 jan. 2016.

CARVALHO, Y. M. Promoção da saúde, práticas corporais e atenção básica. Revista

Brasileira Saúde da Família. Brasília, p. 33-45, 2006.

CARVALHO, Y.M. Práticas corporais e comunidade: um projeto de educação física no Centro de Saúde Escola Samuel B. Pessoa 
(Universidade de São Paulo). In: FRAGA, A. B. e WACHS, F. (Org.). Educação física e saúde coletiva: políticas de formação e perspectivas de intervenção. Porto Alegre: Editora da UFRGS, p. 63-72, 2007.

CARVALHO, Y.M.; FREITAS, F.F. Atividade física, saúde e comunidade. Cadernos de Saúde Coletiva, Rio de Janeiro, v. 14, n. 3, p. 489-505, jul./set., 2006.

\section{CÉSAR, M. S. Práticas corporais na estratégia de saúde da} família: significados do Lian Gong na visão dos usuários de uma comunidade em Porto Alegre. 2012. 37 f. Trabalho de Conclusão de Curso (Curso de Educação Física) Universidade Federal do Rio Grande do Sul, Porto Alegre, 2012.

CONCEIÇÃO, M. R.; MORAES, C. C.; FRANCHINI, M. L. Políticas públicas de saúde, atenção primária e interdisciplinaridade - A produção de cuidado nas práticas corporais. Cadernos de educação, saúde e fisioterapia, v. 1, n. 1, 2014. Disponível em: http:// revista.redeunida.org.br/ojs/index.php/cadernos-educacao-saude-fisioterapia/article/view/5/7. Acesso em: 10 dez. 2015.

\section{DAHLKE, A. P. Práticas corporais em uma Unidade Básica de} Saúde: relato de experiência de uma estudante de Educação Física junto a um grupo de convivência dedicado à vida saudável. 41 f. Monografia. Escola de Educação Física, Curso de Educação Física: Bacharelado, Universidade Federal do Rio Grande do Sul, Porto Alegre, 2014. Disponível em: http://www.lume.ufrgs.br/handle/10183/116105. Acesso em: 10 dez. 2015.

FALCI, D. M.; BELISÁRIO, S. A. A inserção do profissional da educação física na atenção primária à saúde e os desafios em sua formação. Interface. Botucatu, v. 17, n. 47, p. 885-899, oct./dec., 2013.

FRAGA, A. B. et al. Curso de extensão em promoção de saúde para gestores do SUS com enfoque no Programa Academia da Saúde. Brasília: CEAD/UnB, 2013. 144p.

FREITAS, F. F. Aprendendo com o "nós": o trabalho em saúde e as PC com base na comunidade. 230 f. Tese (Doutorado em 
Pedagogia do Movimento Humano) - Escola de Educação Física e Esporte, Universidade de São Paulo, São Paulo, 2012. Disponível em: http://www.teses.usp.br/teses/disponiveis/39/39133/tde07122012-150234/. Acesso em: 2016-01-29.

GIULIANO, A. F. Uma vida boa é uma vida sem dor: percepções das participantes do Grupo Regular de Exercícios Posturais de uma Unidade Básica de Saúde de Porto Alegre. 43 f. Trabalho de Conclusão de Curso. Escola de Educação Física, Curso de Educação Física: Licenciatura, Universidade Federal do Rio Grande do Sul, Porto Alegre, 2012. Disponível em: http://www.lume.ufrgs. br/bitstream/handle/10183/70316/000875709.pdf?sequence=1. Acesso em: 27 jan. 2016.

GORSKI, G. M.; PEREIRA, D. L. Profissional de Educação Física: interdisciplinaridade no Programa Saúde da Família. Revista Ciências em Extensão. v. 9, n. 3, p. 72-81, 2013. Disponível em: http://200.145.6.204/index.php/revista_proex/article/view/869. Acesso em: 23 jan. 2016.

MARCONDES, R. As práticas corporais no serviço público de saúde: uma aproximação entre a educação física e a saúde coletiva. 208 f. Dissertação (Mestrado em Pedagogia do Movimento Humano) - Escola de Educação Física e Esporte, Universidade de São Paulo, São Paulo, 2007. Disponível em: http://www.teses.usp. br/teses/disponiveis/39/39133/tde-19122007-091901/. Acesso em: 2016-01-26.

\section{MEDEIROS, T. F. O Profissional de Educação Física nos}

Núcleos de Apoio à Saúde da Família no Brasil: práticas de Educação em Saúde. 69 f. Trabalho de Conclusão de Curso. Curso de Graduação em Educação Física, Centro de Desportos, Universidade Federal de Santa Catarina, 2013. Disponível em: https://repositorio.ufsc.br/handle/123456789/103773?show=full?. Acesso em: 27 jan. 2016.

MENDES, M. I. B. S. Cenários lúdicos em unidades de saúde da família. Natal: IFRN, 2010. Disponível em: http://vitormarinho. ufsc.br/jspui/bitstream/123456789/241/1/cenariosLudicosUnida- 
desSaudeFamiliaReflexoesPerspectivas.pdf. Acesso em: 28 jan. 2016.

MENDES, V. M. As prática corporais e a clínica ampliada: a educação física na atenção básica. 184 f. Dissertação (Mestrado em Pedagogia do Movimento Humano) - Escola de Educação Física e Esporte, Universidade de São Paulo, São Paulo, 2013. Disponível em: http://www.teses.usp.br/teses/disponiveis/39/39133/tde23052013-121424/. Acesso em: 10 dez. 2015.

MENDONÇA, A. M. Promoção da saúde e processo de trabaIho dos profissionais de educação física do Núcleo de Apoio à Saúde da Família - NASF. 153 f. Dissertação (Mestrado em Saúde Coletiva) - Centro de Ciências da Saúde, Universidade Estadual de Londrina, Paraná, 2012. Disponível em: http://www. uel.br/pos/saudecoletiva/Mestrado/diss/118.pdf. Acesso em: 30 jan. 2016.

MORAIS, O. N. P. Grupos de idosos: atuação da psicogerontologia no enfoque preventivo. Psicologia: ciência e profissão, Brasília, v. 29, n. 4, p. 846-855, 2009. Disponível em: http://pepsic.bvsalud. org/pdf/pcp/v29n4/v29n4a14.pdf. Acesso em: 10 dez. 2015.

NASCIMENTO, R. G. et al. Fisioterapia gerontológica na atenção primária à saúde: uma experiência na região norte. Ciência \& Saúde, v. 6, n. 3, p. 222-228, 2013. Disponível em: http:// revistaseletronicas.pucrs.br/ojs/index.php/faenfi/article/ view/13889/10731. Acesso em: 26 jan. 2016.

SILVA, A. L. F. et al. Educação física na atenção primária à saúde em Sobral-Ceará: desenhando saberes e fazeres integralizados. Sanare: Revista de Políticas Públicas, Sobral, v. 8, n. 2, p. 63-72, 2009. Disponível em: http://sanare.emnuvens.com.br/sanare/ article/view/20/16. Acesso em: 10 dez. 2015.

SILVA, A. M.; DAMIANI, I. R. As práticas corporais em foco: a análise da experiência em questão. In: SILVA, A. M.; DAMIANI, I. R. (Org.). Práticas Corporais. Florianópolis: Nauemblu Ciência e Arte, v. 3, p. 187-207, 2005. 
SKOWRONSKI, M. Educação física e saúde: a mobilização de saberes para atuação nos polos do Programa Academia da Saúde no Brasil. 130 f. Dissertação (Mestrado) - Programa de Pós-Graduação em Ciências do Movimento Humano, Escola de Educação Física, Universidade Federal do Rio Grande do Sul, Porto Alegre, 2014.

TORRES, G. S. Educação Física na estratégia de saúde da família: relato de experiência sobre as atividades na Unidade de Saúde da Família Rincão. 47 f. Trabalho de Conclusão de Curso, Escola de Educação Física. Curso de Educação Física: Licenciatura, Universidade Federal do Rio Grande do Sul, Porto Alegre, 2011. Disponível em: http://www.lume.ufrgs.br/handle/10183/32278. Acesso em: 10 dez. 2015.

VUADEN, F. C. Grupo qualidade de vida: a importância da atividade física na estratégia de saúde da família. 29 f. Trabalho de Conclusão de Curso, Escola de Educação Física. Curso de Educação Física: Licenciatura, Universidade Federal do Rio Grande do Sul, Porto Alegre, 2012. Disponível em: http://www. lume.ufrgs.br/handle/10183/56770. Acesso em: 26 jan. 2016.

WARSCHAUER, M. et al. As escolhas das práticas corporais e dos profissionais que as conduzem nas unidades básicas de saúde do Distrito Butantã-SP. Anais do XV Congresso Brasileiro de Ciências do Esporte. Recife: Edupe, 2007. p. 1-7. Disponível em: http://www.cbce.org.br/docs/cd/resumos/003.pdf. Acesso em: 17 abr. 2016.

WARSCHAUER, M.; D'URSO, L. Ambiência e formação de grupo em programas de caminhada. Saúde e Sociedade, São Paulo, v. 18, n. 2, p. 104-107, 2009. Disponível em: http://www.revistas.usp.br/ sausoc/article/view/29586/31453. Acesso em: 10 dez. 2015. 


\section{Notas}

\section{Publisher}

Universidade Federal de Goiás. Faculdade de Educação Física e Dança. Publicação no Portal de Periódicos UFG. As ideias expressadas neste artigo são de responsabilidade de seus autores, não representando, necessariamente, a opinião dos editores ou da universidade. 